\title{
Effect of Mono and Di-rhamnolipids on Biofilms Pre-formed by Bacillus subtilis BBK006
}

\author{
Mayri A. Díaz De Rienzo ${ }^{1}$ Peter J. Martin ${ }^{1}$
}

Received: 14 January 2016/ Accepted: 12 March 2016/Published online: 25 April 2016

(c) The Author(s) 2016. This article is published with open access at Springerlink.com

\begin{abstract}
Different microbial inhibition strategies based on the planktonic bacterial physiology have been known to have limited efficacy on the growth of biofilms communities. This problem can be exacerbated by the emergence of increasingly resistant clinical strains. Biosurfactants have merited renewed interest in both clinical and hygienic sectors due to their potential to disperse microbial biofilms. In this work, we explore the aspects of Bacillus subtilis BBK006 biofilms and examine the contribution of biologically derived surface-active agents (rhamnolipids) to the disruption or inhibition of microbial biofilms produced by Bacillus subtilis BBK006. The ability of mono-rhamnolipids (Rha- $\left.\mathrm{C}_{10}-\mathrm{C}_{10}\right)$ produced by Pseudomonas aeruginosa ATCC 9027 and the di-rhamnolipids (RhaRha- $\mathrm{C}_{14}-\mathrm{C}_{14}$ ) produced by Burkholderia thailandensis E264, and phosphate-buffered saline to disrupt biofilm of Bacillus subtilis BBK006 was evaluated. The biofilm produced by Bacillus subtilis BBK006 was more sensitive to the di-rhamnolipids $(0.4 \mathrm{~g} / \mathrm{L})$ produced by Burkholderia thailandensis than the mono-rhamnolipids $(0.4 \mathrm{~g} / \mathrm{L})$ produced by Pseudomonas aeruginosa ATCC 9027. Rhamnolipids are biologically produced compounds safe for human use. This makes them ideal candidates for use in new generations of bacterial dispersal agents and useful for use as adjuvants for existing microbial suppression or eradication strategies.
\end{abstract}

Mayri A. Díaz De Rienzo

mayri.diazderienzo@manchester.ac.uk

1 School of Chemical Engineering and Analytical Science, The University of Manchester, Manchester, UK

\section{Introduction}

Biofilms are communities of surface-associated microbial cells enclosed in an extracellular polymeric substance (EPS) matrix. Microbial biofilms represent a different bacterial physiology constituted by a multicellular phenotype which is (generally) very different from planktonic bacteria. Biofilms have been implicated in chronic infections [9]. In the biofilm physiology, these pathogens are several orders of magnitude more resistant to disruption (or killing) by antibiotics than their planktonic counterparts of the same species $[12,13,15]$. The recent advances on biofilm research have enabled researchers to develop more effective bacterial inhibition strategies; currently, there are two main ones [3]: the first is based on the formulation of new antibiofilm molecules and the second the construction of biofilm-resistant surfaces [18].

Biosurfactants are amphiphilic compounds produced on living surfaces, mostly on microbial cells [16]. Biosurfactants have long been reported as molecules with several applications in the industry: detergents, textiles, and with potential applications in environmental and biomedical related areas [8], and more recently as promising candidates for the inhibition of microbial biofilms with anti-adhesive and disruptors properties [7]. Rhamnolipid is a glycolipid biosurfactant constituted of di- or mono-rhamnose sugars attached to a fatty acid chain. These biosurfactants were previously reported as antibacterial agents against $S$. aureus, Bacillus sp, and Klebsiella pneumoniae $[4,7,8,11]$. One of the hypotheses proposed for the biofilm inhibition by rhamnolipids is that they could be involved in the removal of extracellular polymeric substances (EPS) and destruction of microcolonies altering the biofilm environment by their surface activity. 
Rhamnolipids were originally isolated from $P$. aeruginosa, analogues were also produced by isolates of Burkholderia thailandensis [10, 21], which has increased the research interest due to its non-pathogenic nature. In this work, we explore the ability of mono-rhamnolipids (Rha- $\mathrm{C}_{10}-\mathrm{C}_{10}$ ) produced by Pseudomonas aeruginosa ATCC 9027 and the di-rhamnolipids (Rha-Rha- $\mathrm{C}_{14}-\mathrm{C}_{14}$ ) produced by Burkholderia thailandensis to disrupt or inhibit microbial biofilms produced by Bacillus subtilis BBK006.

\section{Materials and Methods}

\section{Microorganisms and Media}

P. aeruginosa ATCC 9027 and B. thailandensis E264 were maintained on nutrient agar slants at $4{ }^{\circ} \mathrm{C}$ in order to minimize biological activity, and were subcultured every month. Each slant was used to obtain a bacterial suspension, with the optical density $(570 \mathrm{~nm})$ adjusted to give $10^{7}$ $\mathrm{CFU} / \mathrm{mL}$ for each of the strains used. The standard medium for the production of rhamnolipids by $P$. aeruginosa ATCC 9027 was PPGAS medium $\left(1 \mathrm{~g} / \mathrm{L} \mathrm{NH} \mathrm{NH}_{4} \mathrm{Cl} 1.5 \mathrm{~g} / \mathrm{L} \mathrm{KCl}\right.$, $19 \mathrm{~g} / \mathrm{L}$ tris- $\mathrm{HCl}, 10 \mathrm{~g} / \mathrm{L}$ peptone, and $0.1 \mathrm{~g} / \mathrm{LMgSO}_{4} \cdot 7 \mathrm{H}_{2}$ $\mathrm{O})$ at $\mathrm{pH}$ 7.4. The fermentation medium contained the same growth medium, with glucose $(0.5 \%)$, as a carbon source. For the production of rhamnolipids by B. thailandensis E264 the media used was nutrient broth (NB) (8 g/L), with glycerol $(20 \mathrm{~g} / \mathrm{L})$. For the antimicrobial assays Bacillus subtilis BBK006 was stored in nutrient broth plus $20 \%$ glycerol at $-80{ }^{\circ} \mathrm{C}$, and used when needed.

\section{Production of Rhamnolipids}

Fermentation units (Electrolab FerMac 360) were used to perform batch cultivation of $P$. aeruginosa ATCC 9027 and B. thailandensis E264. Microorganisms used in this study were aerobically (0.5 VVM) incubated in PPGAS medium and nutrient broth, at 37 and $30^{\circ} \mathrm{C}$, respectively, at $400 \mathrm{rpm}$ speed for $72 \mathrm{~h}$ in the case of $P$. aeruginosa ATCC 9027 and $120 \mathrm{~h}$ for B. thailandensis E264.

\section{Downstream Process for the Purification of Rhamnolipids}

A continuous foam fractionation system in stripping mode was used as a downstream process. $4 \mathrm{~L}$ of rhamnolipid fermentation broth was fed into the top of the straight section of a "J"-shaped glass column of diameter, $D$, $50 \mathrm{~mm}$ and exposed height, $H, 350 \mathrm{~mm}$ via a peristalsis pump, and a metal tube distributor at a feed flow rate of

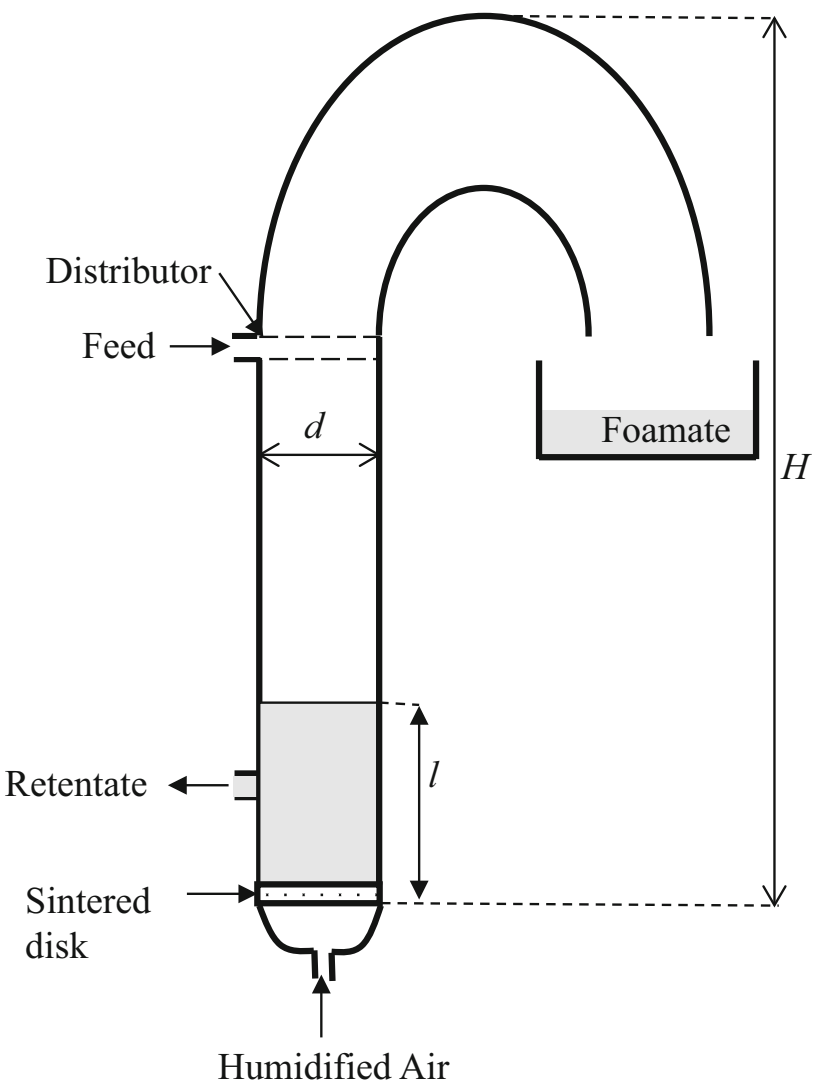

Fig. 1 Schematic diagram of foam fractionation experimental setup $15 \mathrm{~mL} \mathrm{~min}{ }^{-1}$. Figure 1 shows the schematic diagram of the foam fractionation column [20]. Humidified air was sparged through a sintered glass disk into a liquid pool creating overflowing foam. The initial composition of the liquid pool at the bottom of the column was the same as the feed and exited the column through an exit port in such a way that a constant liquid level of $100 \pm 10 \mathrm{~mm}$ was maintained throughout the experiment. The enriched overflowing foam was collected at the open end of the " $\mathrm{J}$ "shaped section. Foam fractionation experiments were performed at different air flow rates for each microorganism. The air flow rate used for $P$. aeruginosa ATCC 9027 was $0.1 \mathrm{~min}^{-1}$ and for $B$. thailandensis E264 the air flow rate used was $1.2 \mathrm{~L} \mathrm{~min}^{-1}$. Each air flow rate was performed in duplicate with fresh fermentation broths for each foam fractionation run.

Foam fractionation was performed for $4 \mathrm{~h}$ to ensure steady-state conditions, and the feed, overflow, and foamate samples were collected every half an hour. The foamate samples were made air tight to prevent evaporation and placed at $4{ }^{\circ} \mathrm{C}$ overnight to collapse foam. The feed, overflow, and diluted foamate samples were analyzed for rhamnolipid concentration after the solvent extraction [17], and the product was used as the disruptor's solutions against Bacillus subtilis BBK006 biofilm. 


\section{Surface Tension Measurements}

Surface tension was evaluated in $10 \mathrm{~mL}$ aliquots of fermented cultures in the absence of biomass, using a Krüss Tensiometer K11 Mk4. Distilled water was used to calibrate the instrument and the measurements were performed in triplicate, using each culture media as a control.

\section{Emulsifying Capacity Determination}

Emulsifying capacity was measured using $5 \mathrm{~mL}$ of kerosene added to $5 \mathrm{~mL}$ of aqueous sample. The mixture is vortex at high speed for $2 \mathrm{~min}$. After $24 \mathrm{~h}$, the height of the stable emulsion layer is measured. The emulsion index $E$ is calculated as the ratio of the height of the emulsion layer and the total height of liquid (Eq. 1).

$E=\frac{h \text { emulsion }}{h \text { total }} \times 100 \%$

\section{Anthrone Assay}

The anthrone assay was used to estimate the concentration of the sugar moiety in the rhamnolipids, in either the freecell culture medium (initial solution), the foamate (collapsed foam), or overflow [20]. Briefly, about $20 \mathrm{mg}$ of anthrone was dissolved in a $70 \%(\mathrm{v} / \mathrm{v})$ sulfuric acid solution with gentle warming. The anthrone reaction (different concentrations were tested) was done by pipetting $0.1 \mathrm{~mL}$ of a test sample into an eppendorf tube. Then, $1 \mathrm{~mL}$ of the anthrone reagent was slowly added into the tube with agitation. After being thoroughly mixed, the tube was stoppered, and was placed in a vigorously boiling water bath for $10 \mathrm{~min}$. After that, the tube was left at room temperature for $30 \mathrm{~min}$. A bluish green solution was achieved, and its absorbance was measured at a wavelength of $625 \mathrm{~nm}$ by using a UV/Vis spectrophotometer (Shimadzu Uvmini-1240). The amount of biosurfactant in the test sample was subsequently calculated in terms of $\mathrm{g} / \mathrm{L}$ of rhamnose in the test sample by using a calibration curve of the colored solution obtained from the reaction between the anthrone reagent and the standard rhamnose in the concentration range of $100-800(\mathrm{~g} / \mathrm{L})$.

\section{ESI-MS Analysis}

For mass analyses, partially purified rhamnolipid preparations (either the free-cell culture medium, the foamate, or overflow) were dissolved in water and characterized by ESI-MS (electrospray ionization-mass spectrometry) using a Waters LCT mass spectrometer in negative-ion mode previously tuned and calibrated on NaF. $20 \mu \mathrm{L}$ was flow injected into a mobile phase consisting of $50 \% \mathrm{ACN}-0.1 \%$ formic acid using a Waters Alliance 1170 HPLC.

\section{Growth of Biofilm in Flow Cells}

Biofilms of Bacillus subtilis BBK006 were allowed to form in a flow cell system. The system comprised a flow cell that served as a growth chamber for the biofilms. The flow cell was supplied with nutrients and oxygen from a medium flask containing NB via a peristaltic pump ( $\mathrm{mL} / \mathrm{h} / \mathrm{channel})$ and spent medium was collected in a waste container. A bubble trapping device confined air bubbles from the tubing which otherwise could disrupt the biofilm structure in the flow cell. After $48 \mathrm{~h}$ of incubation at $30{ }^{\circ} \mathrm{C}$, the medium was replaced with different treatments [phosphatebuffered saline (PBS) buffer 1X, mono-rhamnolipids $0.4 \mathrm{~g} /$ $\mathrm{L}$, and di-rhamnolipids $0.4 \mathrm{~g} / \mathrm{L}$ ] for $30 \mathrm{~min}$. After treatment, the cells were stained with LIVE/DEAD ${ }^{\circledR}$ BacLight ${ }^{\mathrm{TM}}$ Kit and observed using a Leica SP5 inverted confocal microscope, providing highly detailed 3D information about the development of microbial biofilms using FiJi [14].

\section{Results and Discussion}

\section{Production of Rhamnolipids}

Pseudomonas aeruginosa ATCC 9027 and Burkholderia thailandensis E264 were able to produce glycolipids biosurfactants under aerobic conditions. After $72 \mathrm{~h}, \mathrm{Pseu}$ domonas aeruginosa ATCC 9027 was able to produce rhamnolipids on PPGAS medium at $37{ }^{\circ} \mathrm{C}$ after $48 \mathrm{~h}$, using glucose $(5 \mathrm{~g} / \mathrm{L})$ as carbon source. On the other hand, Burkholderia thailandensis E264 was able to produce rhamnolipids on nutrient broth using glycerol $(20 \mathrm{~g} / \mathrm{L})$ as carbon source.

All the rhamnolipids production in $P$. aeruginosa is associated to their virulence factors, which are regulated via quorum sensing (QS) system (nevertheless, it has not been demonstrated, yet the presence of a QS system on $B$. thailandensis is linked to the presence of $r h l A, r h l B$, and $r h l C)$. This might be one of the reasons affecting the production yields for both rhamnolipids types (Table 1). In Pseudomonas case, the production is associated to the growing, while in $B$. thailandensis could be a metabolite that is been produced along with another proteins like efflux pumps and transporter, whose genes are in the $r h l$ cluster.

The rhamnolipids produced by B. thailandensis E264 reduced the surface tension up to $32 \mathrm{mN} / \mathrm{m}$, in contrast with $P$. aeruginosa ATCC rhamnolipids where the surface tension was reduced up to $24 \mathrm{mN} / \mathrm{m}$. This could be an 
Table 1 P. aeruginosa ATCC 9027 and B. thailandensis E264 biomass and rhamnolipid production yields

\begin{tabular}{llllll}
\hline Microorganisms & \multicolumn{2}{l}{ Glycerol $20 \mathrm{~g} / \mathrm{L}$} & & \multicolumn{2}{l}{ Glucose $5 \mathrm{~g} / \mathrm{L}$} \\
\cline { 2 - 3 } \cline { 6 - 7 } & $\mathrm{X}(\mathrm{g} / \mathrm{L})$ & $\mathrm{Y}_{\mathrm{p} / \mathrm{s}}$ & & $\mathrm{X}(\mathrm{g} / \mathrm{L})$ & $\mathrm{Y}_{\mathrm{p} / \mathrm{s}}$ \\
\hline P. aeruginosa ATCC 9027 & - & - & & 2.5 & 0.32 \\
B. thailandensis E264 & 9.5 & 0.025 & - & - \\
\hline
\end{tabular}

- Not detected

indication of both molecules been structurally different, and resulting in different hydrophilic-lipophilic balance (HLB) with different values. These values are similar to those previously reported for $P$. aeruginosa sp. with values between 25 and $30 \mathrm{mN} / \mathrm{m}$ [2]. For $B$. thailandensis, the ability to produce rhamnolipids was first in 2009 [10] where the reduction of the surface tension was $42 \mathrm{mN} / \mathrm{m}$.

The different microorganisms were assessed for their ability to form stable emulsions on the supernatant phase, and the results show a $65 \%$ of emulsion for rhamnolipids produced by $P$. aeruginosa ATCC 9027 and a $42 \%$ for those produced by $B$. thailandensis E264 after 11 days of cultivation.

Foam fractionation studies in continuous mode were used for the recovery of the excreted biosurfactant from the cell-free culture medium produced by both microorganisms. The molecular and surface chemistry properties of the feed, foamate, and overflow were analyzed by ESI-MS.

Foam fractionation separation performance was evaluated in terms of recovery and enrichment. Figure 2 shows the recovery and enrichment variation with increasing air flow rate for rhamnolipids produced by $P$. aeruginosa ATCC 9027 and B. thailandensis E264. The results show that the recovery and enrichment of rhamnolipids produced by $P$. aeruginosa ATCC 9027 increased and decreased, respectively, with increasing air flow rate.

This is as expected for a single component system where with increasing air flow rate, the residence time of the bubbles in the column decreases. These different recovery and enrichment behaviors might suggest the presence of other surface-active species in the fermentation broth other than the rhamnolipids produced.

ESI-MS analysis revealed the presence of different congeners of rhamnolipids produced by each microorganism. In the case of B. thailandensis E264, a dominant peak in the ESI-MS was shown a pseudomolecular ion of $\mathrm{m} / \mathrm{z} 761$ in negative-ion mode (Fig. 3a), a value that is compatible with a 2- $O$ - $\alpha$-L-rhamnopyranosyl- $\alpha$-L-rhamnopyranosyl- $\beta$-hydroxytetradecanoyl- $\beta$-ydroxytetradecanoate (Rha-Rha$\mathrm{C}_{14}-\mathrm{C}_{14}$ ), with a molecular weight of $762 \mathrm{Da}$, that it has been previously reported from $B$. pseudomallei and B. plantarii and $B$. thailandensis itself $(6,10)$.

To confirm the rhamnolipid production by $P$. aeruginosa ATCC 9027, the same ESI-MS method was used. The presence of the mono-rhamnolipid rhamnosyl-3-hydroxydecanoyl-3-hydroxydecanoate Rha- $\mathrm{C}_{10}-\mathrm{C}_{10}$ was revealed as a predominant peak of $m / z 503$ (Fig. 3b), in accordance with the previous reports where it has been widely studied and surveyed for the past decade $[1,16,22]$.

The analysis of B. thailandensis E264 cultures revealed long chain rhamnolipids, with a different HLB from the one that $P$. aeruginosa ATCC 9027 was able to produce (under the conditions tested in this work), which supports the hypothesis presented where both microorganisms produce different molecules that could have different applications from a biotechnology point of view.
Fig. 2 Recovery and enrichment of Rha- $\mathrm{C}_{10}-\mathrm{C}_{10}$ and Rha-Rha- $\mathrm{C}_{14}-\mathrm{C}_{14}$ with increasing air flow

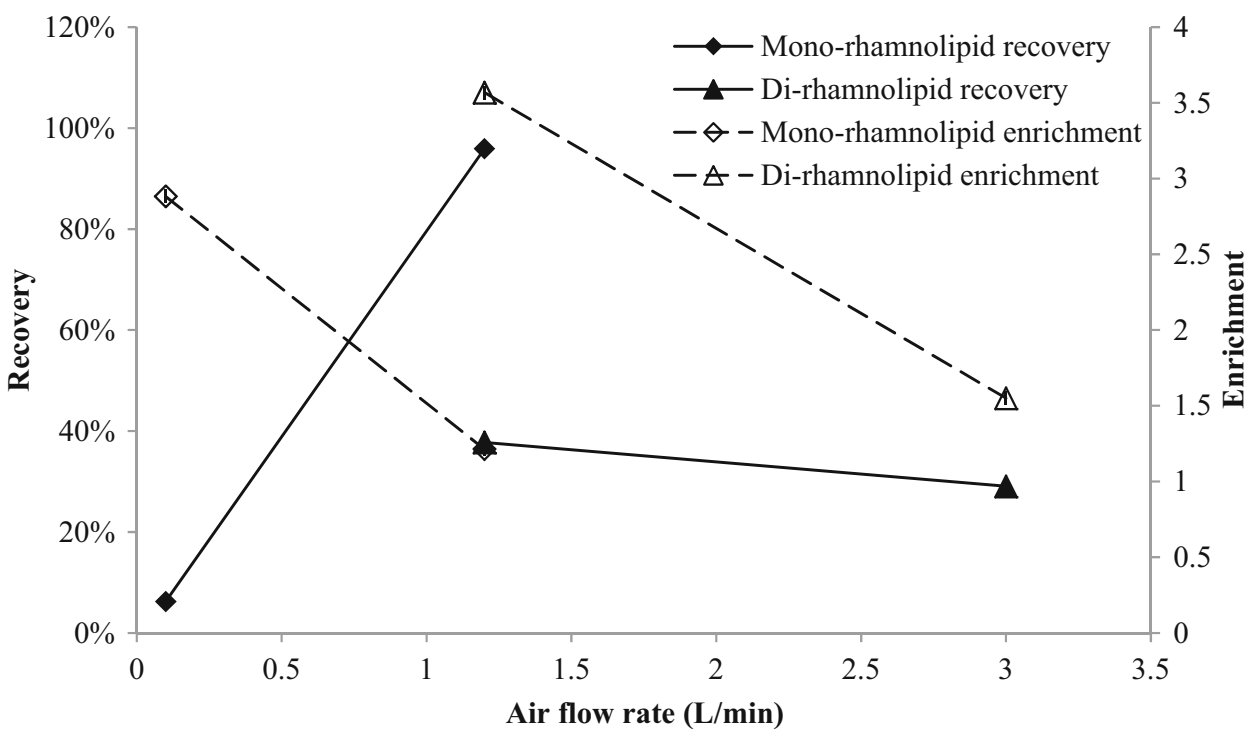


A MDR S8 $20150506 \_$RAS_009 23 (0.382) AM (Cen.4.20.00.Ar.5000.0.0.00.1.00): Sb (15.10.00): Sm (Mn, 3x.500): Cm (20:29
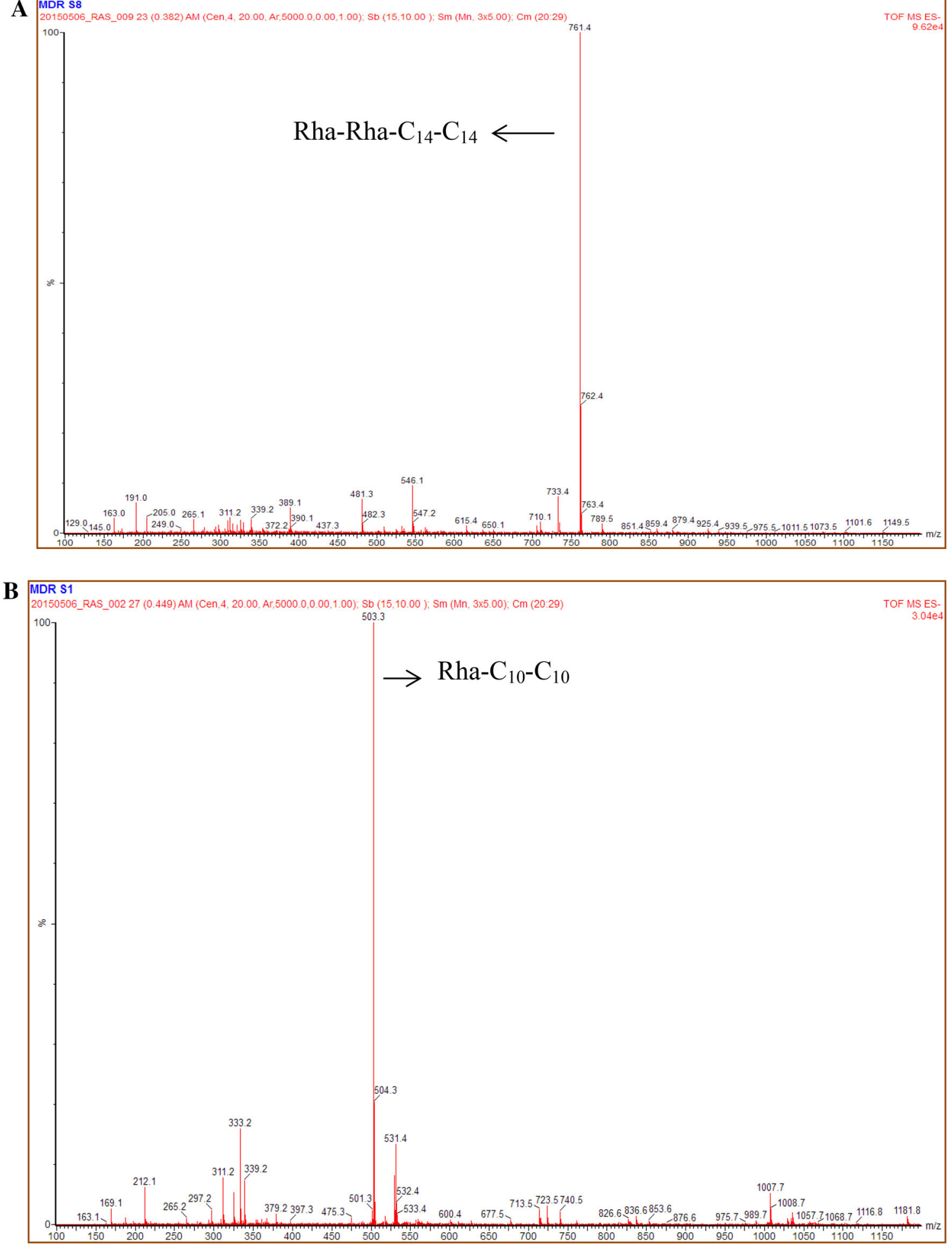

Fig. 3 ESI-MS analysis. Spectrum of partially purified extracts from fermented cells of a B. thailandensis E264 and b P. aeruginosa ATCC 9027 (Rha: rhamnose molecules) in the feed fraction 
Fig. 4 Confocal microscopy micrographs. a Threedimensional (left panels) and b orthogonal reconstructions (right panels) of the biofilm formed by Bacillus subtilis BBK066. The pictures refer to the various experimental conditions as indicated on the left. The fluorescence is associated with live (green) and dead (red) cells, respectively. Scale bars represent $30 \mu \mathrm{m}$ as indicated in micrographs (Color figure online)

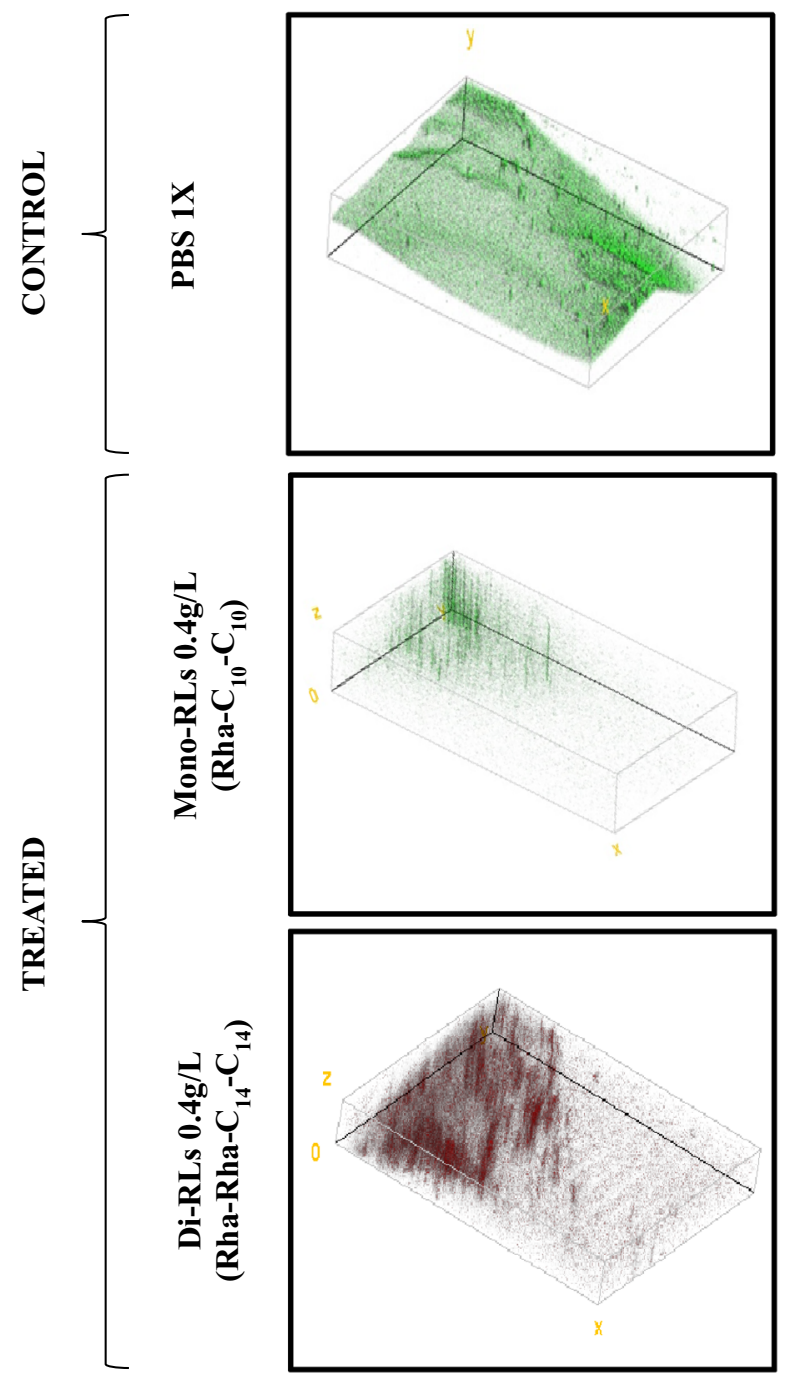

(A)
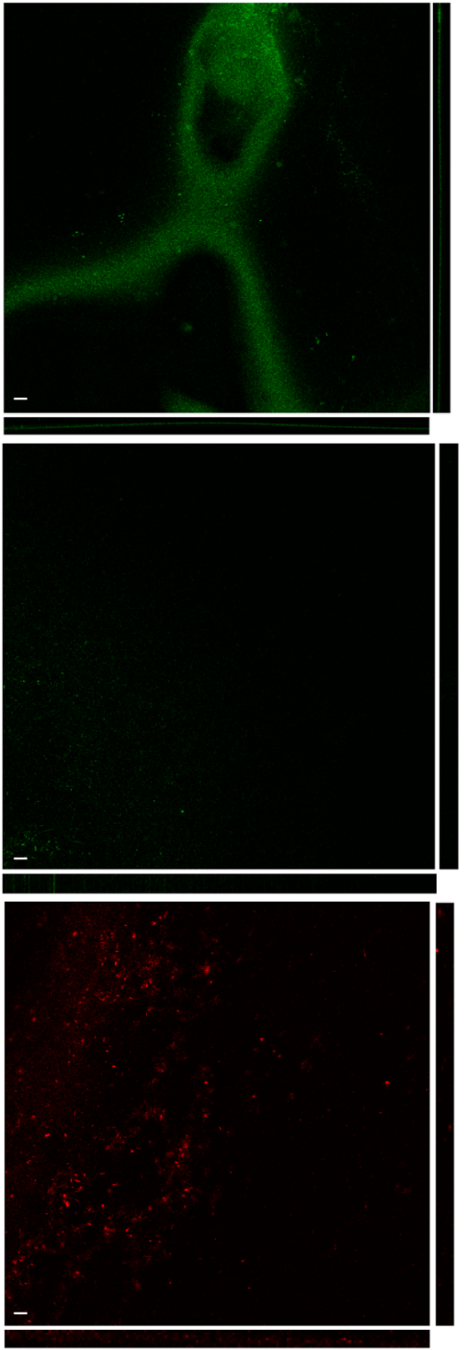

(B)

\section{Effect of Different Rhamnolipids on Pre-formed Biofilms by Bacillus subtilis BBK006 in Flow Cell}

It has been reported before [7] that pre-formed biofilms by Bacillus subtilis can be disrupted by sophorolipids. In a recent work [8], studies demonstrated the effect of rhamnolipids against biofilms formed by selected gram-negative and gram-positive bacteria on static conditions; however, the effect of specific rhamnolipids congeners on biofilms formed by Bacillus subtilis has not been reported yet.

In this work, we evaluated the effect of Rha- $\mathrm{C}_{10}-\mathrm{C}_{10}$, Rha-Rha- $\mathrm{C}_{14}-\mathrm{C}_{14}$ and the ionic surfactant SDS on Bacillus subtilis BBK066 biofilms developed on a flow cell system. The cells were stained with LIVE/DEAD ${ }^{\circledR}$ BacLight $^{\mathrm{TM}}$ Kit, and confocal microscopy was used to analyze the data. Biofilm was grown for 2 days in continuous flow mode in a flow cell channel. Before the addition of each treatment, a developed biofilm was observed (data not shown); the thickness of the biofilm was about $15 \mu \mathrm{m}$. After $30 \mathrm{~min}$ of each treatment (PBS 1X, SDS $0.4 \mathrm{~g} / \mathrm{L}$, Rha- $\mathrm{C}_{10}-\mathrm{C}_{10} 0.4 \mathrm{~g} / \mathrm{L}$, and Rha-Rha- $\mathrm{C}_{14}-\mathrm{C}_{14} 0.4 \mathrm{~g} / \mathrm{L}$ ), different results were observed.

The SDS has a remarkable effect on Bacillus subtilis BBK006 biofilm disruption at $0.04 \mathrm{~g} / \mathrm{L}$ (data not shown), in comparison to those treated with PBS $1 \mathrm{X}$ where all the cells were well established and viable (Fig. 4). However, when the cells were treated with $\mathrm{Rha}-\mathrm{C}_{10}-\mathrm{C}_{10}$ or RhaRha- $\mathrm{C}_{14}-\mathrm{C}_{14}$, the disruption is appreciated. In an interesting way, the cells treated with Rha-Rha- $\mathrm{C}_{14}-\mathrm{C}_{14}$ seem to be showing an inhibitory effect in the biofilm disruption judged by the red stain observed.

The results we have obtained demonstrated the inhibitory effect of rhamnolipids on pre-formed Bacillus subtilis BBK066 biofilms, similar to those reported by Davey [5]. In the same context, Dusane et al. [11] reported the effect of rhamnolipids on pre-formed biofilms of Bacillus pumilus 
from the marine environment, resulting in a dispersal at sub-MIC concentrations and confirming the ability to disrupt them. The effect of rhamnolipids on biofilms formed by gram-positive microorganisms relies on the ability in the removal of the matrix components to facilitate the detachment of the cells to the surface. Different congeners would possibly have different impacts on the cell surfaces where the overall charge and the length of the fatty acid chain will not just allow the removal of the matrix components but the penetration on the cell membrane with a bactericidal effect. Nevertheless, further work is required to confirm this hypothesis, taking into account that the effect in most of the cases would be species-specific. In addition, it will be worth to evaluate the combination between rhamnolipids and proteins, or any other molecule that could lead to find a specific strategy to eradicate biofilms of different microorganisms, either on static or continuous systems.

Acknowledgments The authors are grateful for financial support from the UK Engineering and Physical Sciences Research Council $(\mathrm{EP} / \mathrm{I} 024905)$ which enabled this work to be conducted.

Open Access This article is distributed under the terms of the Creative Commons Attribution 4.0 International License (http://crea tivecommons.org/licenses/by/4.0/), which permits unrestricted use, distribution, and reproduction in any medium, provided you give appropriate credit to the original author(s) and the source, provide a link to the Creative Commons license, and indicate if changes were made.

\section{References}

1. Al-Tahhan R, Sandrin T, Bodour A, Maier R (2000) Rhamnolipid-induced removal of lipopolysaccharide from Pseudomonas aeruginosa: effect on cell surface properties and interaction with hydrophobic substrates. Appl Environ Microbiol 66(8):32623268

2. Andrä J, Rademann J, Howe J, Koch MHJ, Heine H, Zähringer U, Brandenburg K (2006) Endotoxin-like properties of a rhamnolipid exotoxin from Burkholderia (Pseudomonas) plantarii: immune cell stimulation and biophysical characterization. Biol Chem 387:301-310

3. Banat IM, Díaz De Rienzo MA, Quinn GA (2014) Microbial biofilms: biosurfactants as antibiofilm agents. Appl Microbiol Biotechnol 98:9915-9929

4. Benincasa M, Abalos A, Oliveira I, Manresa A (2004) Chemical structure, surface properties and biological activities of the biosurfactant produced by Pseudomonas aeruginosa LBI from soapstock. Antonie Van Leeuwenhoek 85:1-8

5. Davey ME, Caiazza NC, O’Toole GA (2003) Rhamnolipid surfactant production affects biofilm architecture in Pseudomonas aeruginosa PAO1. J Bacteriol 185:1027-1036

6. Déziel E, Lépine F, Dennie D, Boismenu D, Mamer OA, Villemur R (1999) Liquid chromatography/mass spectrometry analysis of mixtures of rhamnolipids produced by Pseudomonas aeruginosa strain 57RP grown on mannitol or naphthalene. Biochim Biophys Acta 1440:244-252

7. Díaz De Rienzo MA, Banat IM, Dolman B, Winterburn J, Martin $P$ (2015) Sophorolipid biosurfactants: possible uses as antibacterial and antibiofilm agent. New Biotechnol 32(6):720-726

8. Díaz De Rienzo MA, Stevenson P, Marchant R, Banat IM (2016) Antibacterial properties of Biosurfactants against selected gram positive and negative bacteria. FEMS Microbiol Lett. doi:10. 1093/femsle/fnv224

9. Dowd SE, Wolcott RD, Sun Y, McKeehan T, Smith E, Rhoads D (2008) Polymicrobial nature of chronic diabetic foot ulcer biofilm infections determined using bacterial Tag encoded FLX amplicon pyrosequencing (bTEFAP). PLoS ONE 3:e3326

10. Dubeau D, Deziel E, Woods DE, Lepine F (2009) Burkholderia thailandensis harbors two identical $r h l$ gene clusters responsible for the biosynthesis of rhamnolipids. BMC Microbiol 9:263

11. Dusane DH, Nancharaiah YV, Zinjarde SS, Venugopalan VP (2010) Rhamnolipid mediated disruption of marine Bacillus pumilus biofilms. Coll Surf B 81:242-248

12. Girard LP, Ceri H, Gibb AP, Olson M, Sepandj F (2010) MIC versus MBEC to determine the antibiotic sensitivity of Staphylococcus aureus in peritoneal dialysis peritonitis. Perit Dial Int 30:652-656

13. Olson ME, Ceri H, Morck DW, Buret AG, Read RR (2002) Biofilm bacteria: formation and comparative susceptibility to antibiotics. Can J Vet Res 66:86-92

14. Schindelin J, Arganda-Carreras I, Frise E, Kaynig V, Longair M, Pietzsch T, Preibisch S, Rueden C, Saalfeld S, Schmid B, Tinevez J, White D, Hartenstein V, Eliceiri K, Tomancak P, Cardona A (2012) Fiji: an open-source platform for biological-image analysis. Nat Methods 9:676-682

15. Sepandj F, Ceri H, Gibb A, Read R, Olson M (2004) Minimum inhibitory concentration (MIC) versus minimum biofilm eliminating concentration (MBEC) in evaluation of antibiotic sensitivity of gramnegative bacilli causing peritonitis. Perit Dial Int 24:65-67

16. Setoodeh P, Jahanmiri A, Eslamloueyan R, Niazi A, Ayatollahi S, Aram F, Mahmoodi M, Hortamani A (2014) Statistical screening of medium components for recombinant production of Pseudomonas aeruginosa ATCC 9027 rhamnolipids by nonpathogenic cell factory Pseudomonas putida KT2440. Mol Biotechnol 56:175-191

17. Silva R, Almeida D, Rufino R, Luna J, Santos V, Sarubbo L (2014) Applications of biosurfactants in the petroleum industry and the remediation of oil spills. Int J Mol Sci 15:12523-12542

18. Smyth TJP, Perfumo A, Marchant R, Banat IM (2010) Isolation and analysis of low molecular weight microbial glycolipids. In: Timmis KN (ed) Handbook of hydrocarbon and lipid microbiology. Springer, Berlin, pp 3705-3724

19. Villa F, Cappitelli F (2013) Plant-derived bioactive compounds at sublethal concentrations: towards smart biocide-free antibiofilm strategies. Phytochem Rev 12:245-254

20. Winterburn JB, Russell AB, Martin PJ (2011) Integrated recirculating foam fractionation for the continuous recovery of biosurfactant from fermenters. Biochem Eng J 54:132-139

21. Yu Y, Kim HS, Chua HH, Lin CH, Sim SH, Lin D, Derr A, Engels R, DeShazer D, Birren B, Nierman WC, Tan P (2006) Genomic patterns of pathogen evolution revealed by comparison of Burkholderia pseudomallei, the causative agent of melioidosis, to avirulent Burkholderia thailandensis. BMC Microbiol 6:46

22. Zhang Y, Miller R (1992) Enhanced octadecane dispersion and biodegradation by a Pseudomonas rhamnolipid surfactant (biosurfactant). Appl Environ Microbiol 58(10):3276-3282 